\title{
Effects of Blood Contamination on Microtensile Bond Strength to Dentin of Three Self-etch Adhesives
}

\author{
SW Chang • BH Cho $\bullet$ RY Lim \\ SH Kyung • DS Park • TS Oh • HM Yoo
}

\section{Clinical Relevance}

When blood contamination occurs during dentin bonding with self-etch adhesives, blood contamination cannot be adequately removed by water rinsing alone.

Seok Woo Chang, DDS, MSD, assistant professor, Department of Conservative Dentistry, The Institute of Oral Health Science, Samsung Medical Center, Sungkyunkwan University School of Medicine, Seoul, Korea

Byeong Hoon Cho, DDS, MSD, PhD, professor, Department of Conservative Dentistry, School of Dentistry, Dental Research Institute, Seoul National University, Seoul, Korea

Ran Yeob Lim, DDS, graduate student, Department of Conservative Dentistry, The Institute of Oral Health Science, Samsung Medical Center, Sungkyunkwan University School of Medicine, Seoul, Korea

Seung Hyun Kyung, DDS, MSD, PhD, associate professor, Department of Orthodontics, The Institute of Oral Health Science, Samsung Medical Center, Sungkyunkwan University School of Medicine, Seoul, Korea

Dong Sung Park, DDS, MSD, PhD, professor, Department of Conservative Dentistry, The Institute of Oral Health Science, Samsung Medical Center, Sungkyunkwan University School of Medicine, Seoul, Korea

Tae Seok Oh, DDS, MSD, PhD, chairperson, professor, Department of Conservative Dentistry, The Institute of Oral Health Science, Samsung Medical Center, Sungkyunkwan University School of Medicine, Seoul, Korea

*Hyun Mi Yoo, DDS, MSD, PhD, associate professor, Department of Conservative Dentistry, The Institute of Oral Health Science, Samsung Medical Center, Sungkyunkwan University School of Medicine, Seoul, Korea *Reprint request: 50 Ilwon-dong, Gangnam-gu, Seoul, Korea,
135-710; e-mail: smcyoo@hanmail.net

DOI: $10.2341 / 09-244-\mathrm{L}$

\section{SUMMARY}

This study evaluated the effects of blood contamination and decontamination methods during different steps of bonding procedures on the microtensile bond strength of two-step self-etch adhesives to dentin. Sixty extracted human molars were ground flat to expose occlusal dentin. The 60 molars were randomly assigned to three groups, each treated with a different twostep self-etch adhesive: Clearfil SE Bond, AdheSE and Tyrian SPE. In turn, these groups were subdivided into five subgroups $(n=20)$, each treated using different experimental conditions as follows: control group-no contamination; contamination group 1-CG1: primer application/ contamination/primer re-application; contamination group 2-CG2: primer application/contamination/wash/dry/primer re-application; contamination group 3-CG3: primer application/adhesive application/light curing/contamination/ adhesive re-application/light curing; contamina- 
tion group 4-CG4: primer application/adhesive application/light curing/contamination/wash/ dry/adhesive re-application/light curing.

Composite buildup was performed using Z250. After 24 hours of storage in distilled water at $37^{\circ} \mathrm{C}$, the bonded specimens were trimmed to an hourglass shape and serially sectioned into slabs with $0.6 \mathrm{~mm}^{2}$ cross-sectional areas. Microtensile bond strengths (MTBS) were assessed for each specimen using a universal testing machine. The data were analyzed by two-way ANOVA followed by a post hoc LSD test. SEM evaluations of the fracture modes were also performed. The contaminated specimens showed lower bond strengths than specimens in the control group $(p<0.05)$, with the exception of CG1 in the Clearfil SE group and CG2 and CG3 in the Tyrian SPE group. Among the three self-etch adhesives, the Tyrian SPE group exhibited a significantly lower average MTBS compared to the Clearfil SE Bond and AdheSE $(p<0.05)$ groups. Based on the results of the current study, it was found that blood contamination reduced the MTBS of all three self-etch adhesives to dentin, and water-rinsing was unable to overcome the effects of blood contamination.

\section{INTRODUCTION}

There is an increasing demand for esthetic restoration in operative dentistry. This has led to the intensive study of adhesive materials. Good adhesion to dental hard tissues is one of the prime prerequisites for resinbased dental composites. ${ }^{1}$ Any contamination of the preparation surface by saliva, blood or gingival crevicular fluid should be avoided in order to achieve a successful and durable bond between the resin composite and the tooth. However, moisture control is difficult in caries located at or near the gingival margin. Rubber dam isolation is difficult to apply in these areas, and contamination of the operating field with blood or saliva is likely to occur. Blood contamination has been reported to decrease bond strength when it occurs after collagen fibers have been exposed by acid etching. ${ }^{2}$

Self-etch adhesives are commonly employed for bonding procedures, because they are user-friendly and yield good clinical results. ${ }^{2-3}$ Self-etch adhesives do not require a separate etch and rinse step, the omission of which reduces technique sensitivity and operating time, as well as reducing the risk of contamination on the etched tooth surface. ${ }^{4}$ It has been hypothesized that self-etch adhesives may be more resistant to saliva contamination because hydrophilic adhesive solutions, specifically acetone- or ethanol-based products, may displace or diffuse through a salivary film to reach the underlying hydroxyapatite or collagen. ${ }^{5}$
Previous studies have investigated the effects of saliva contamination ${ }^{4-12}$ and blood contamination ${ }^{6,13-20}$ on bonding between resin composites and tooth surfaces. However, there have been relatively few studies $^{6,13-15,20}$ on the effects of blood contamination on dentin bond strength. Furthermore, these studies used either primary teeth as the subjects, ${ }_{13}^{13}$ etch and rinse ${ }^{6,15,20}$ or onebottle self-etch adhesives. ${ }^{14}$ The effects of blood contamination on the bond strength of two-step self-etch adhesives has rarely been studied ${ }^{21}$ despite the frequent clinical use of these adhesives. For this reason, the authors of the current study investigated the effects of blood contamination on the microtensile bond strength (MTBS) of two-step self-etch adhesives using three commercial materials: Clearfil SE (Kuraray, Okayama, Japan), AdheSE (Ivoclar Vivadent, Schaan, Liechtenstein) and Tyrian SPE (BISCO, Inc, Schaumburg, IL, USA), which are mild, moderate and strong self-etching adhesives, respectively. ${ }^{22}$ Many previous studies ${ }^{23-26}$ have investigated the bond strengths of these two-step self-etch adhesives. However, as best as the authors can determine, a comparative study of the effects of blood contamination on MTBS of these three self-etch adhesives has never been conducted.

The current study evaluated the effects of blood contamination on the MTBS of three two-step self-etch adhesives to dentin. The null hypothesis of this study was that blood contamination would not have an adverse effect on the MTBS of two-step self-etch adhesives.

\section{METHODS AND MATERIALS}

The materials used in this study included three commercial two-step self-etch adhesives: Clearfil SE Bond (Kuraray, Okayama, Japan), AdheSE (Ivoclar Vivadent) and Tyrian SPE/One-Step Plus (BISCO, Inc). The composition of the self-etch adhesives used in this study are listed in Table 1. Filtek Z-250 (3M ESPE, St Paul, MN, USA) was used as a restorative dental composite.

All the experimental procedures were performed by a single operator to reduce inter-operator variation. Sixty extracted, caries-free human molars were stored in distilled water containing $0.5 \%$ thymol. After removing the enamel layer from each tooth, the exposed dentin was ground with 600 grit $\mathrm{SiC}$ paper under running water to provide a standard experimental condition. Fresh human capillary blood was collected from one participant at the same time that the specimens were prepared. The 60 molars were randomly assigned to one of three experimental groups corresponding to the three self-etch adhesives tested. The 20 molars in each self-etch adhesive group were further divided among five experimental groups per adhesive type as follows: 


\begin{tabular}{|c|c|c|c|}
\hline Adhesive & Manufacturer & Composition & Techniques \\
\hline Clearfil SE Bond & $\begin{array}{l}\text { Kuraray Medical Inc, } \\
\text { Okayama, Japan }\end{array}$ & $\begin{array}{l}\text { self-etching primer: 10- methacryloxydecyl } \\
\text { dihydrogen phosphate (MDP), 2- hydroxyethyl } \\
\text { methacrylate (HEMA), hydrophilic dimethacrylate, } \\
\text { di-camphorquinone, N,N-diethanol-p-toluidine, water } \\
\text { bonding agent: 10- methacryloxydecyl dihydrogen } \\
\text { phosphate (MDP), bis-phenol A diglycidylmethacrylate } \\
\text { (Bis-GMA), 2- hydroxyethyl methacrylate (HEMA), } \\
\text { hydrophobic dimethacrylate, di-camphorquinone, } \\
\text { N,N-diethanol-p-toluidine, silanated colloidal silica }\end{array}$ & $\begin{array}{l}\text { apply primer } 20 \\
\text { seconds, air dry } \\
\text { apply, air dry } \\
\text { light cure } \\
10 \text { seconds }\end{array}$ \\
\hline AdheSE & $\begin{array}{l}\text { Ivoclar Vivadent AG, } \\
\text { Schaan, Liechtenstein }\end{array}$ & $\begin{array}{l}\text { self-etching primer: dimethacrylate, phosphoric } \\
\text { acid acrylate, initiators and stabilizers in an aqueous } \\
\text { solution } \\
\text { bonding agent: HEMA, dimethacrylate, silicon dioxide, } \\
\text { initiators and stabilizers }\end{array}$ & $\begin{array}{l}\text { apply primer } 30 \\
\text { seconds, air dry } \\
\text { apply, air dry } \\
\text { light cure } \\
10 \text { seconds }\end{array}$ \\
\hline Tyrian SPE & $\begin{array}{l}\text { BISCO, Inc, } \\
\text { Schaumburg, IL, USA }\end{array}$ & $\begin{array}{l}\text { primer A: thymol blue, ethanol and water } \\
\text { primer B: AMPS, Bis MEP, TPO and ethanol }\end{array}$ & $\begin{array}{l}\text { primer } A+B \\
\text { apply primer } 20 \\
\text { seconds, air dry }\end{array}$ \\
\hline One-step Plus & & $\begin{array}{l}\text { bonding agent: bisphenyl dimethacrylate, hydroxyethyl } \\
\text { methacrylate, acetone }\end{array}$ & $\begin{array}{l}\text { apply, air dry light } \\
\text { cure } 10 \text { seconds }\end{array}$ \\
\hline
\end{tabular}

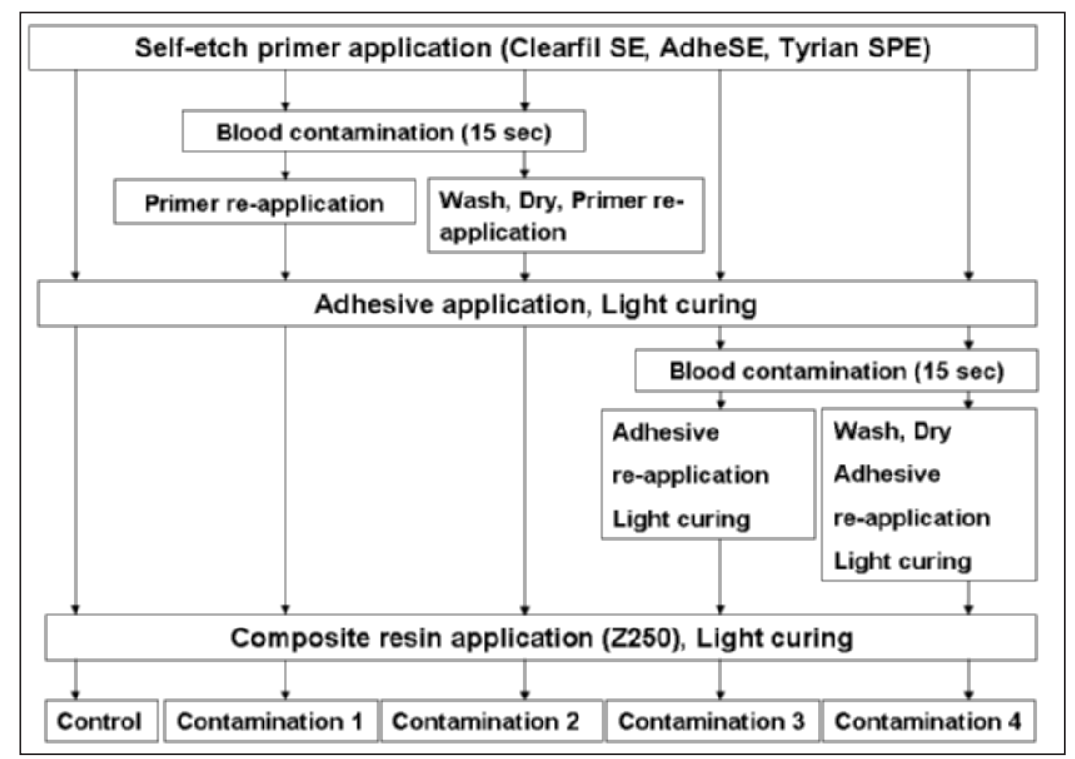

Figure 1: Schematic representation of the experimental design. The steps at which blood contamination occurred during the bonding procedure and the methods of decontamination are described.

Control group ( $\mathrm{n}=20$ ): In this group, there was no blood contamination. Self-etch primers and adhesives were applied to the dentin of each specimen according to the manufacturer's instructions. The adhesive was light-cured for 10 seconds using a visible light-curing unit (Optilux402, Demetron/Kerr, Danbury, CT, USA).

Contamination group 1 (CG1: $n=20$ ): The self-etch primer was applied in the same manner as the control group. Before applying the adhesive, the primer-treated surface was contaminated with fresh blood for 15 seconds using a microbrush. The self-etch primer was then reapplied to the contaminated surface with a gen- tle scrubbing motion until no blood was observed. Thereafter, the adhesive was applied and light-cured for 10 seconds (Optilux402, Demetron/Kerr).

Contamination group 2 (CG2: $n=20)$ : The methods of self-etch primer application and blood contamination were the same as in CG1. After contamination, the blood was rinsed for 10 seconds with a water stream from an airwater syringe. A gentle puff of air was then applied for two seconds to dry the surface. Care was taken to not desiccate the surface. Thereafter, the adhesive was applied and lightcured in the same manner as in CG1.

Contamination group 3 (CG3: $\mathrm{n}=20$ ): The selfetch primer and adhesive were applied to the dentin and light cured in the same manner as the control group. The surface was then contaminated with fresh blood for 15 seconds using a microbrush. Thereafter, adhesive was reapplied to the blood-contaminated surface until no blood was observed. It was then lightcured for 10 seconds (Optilux 402, Demetron/ Kerr).

Contamination group 4 (CG4: $\mathrm{n}=20$ ): Self-etch primer application, light-curing and blood contamination were conducted in the same manner as in CG3. The blood was then rinsed for 10 seconds with a water stream from an air-water syringe and dried with a gentle puff of air for two seconds. Thereafter, the adhesive was applied and light-cured in the same manner as in CG3.

These experimental designs are illustrated in Figure 1. 
After light-curing the adhesive on each specimen, resin composite was applied to the bonding surface in three increments to a final thickness of $3.6 \mathrm{~mm}$. Each increment was light-cured for 20 seconds with a visible light-curing unit (Optilux 402, Demetron/Kerr). The light intensity was periodically measured during the experimental procedures using a radiometer (Model 100 , Demetron/Kerr). It was confirmed that the lightcuring unit worked in the range from 520 to 560 $\mathrm{mW} / \mathrm{cm}^{2}$.

All bonded specimens were stored in distilled water for 24 hours at $37^{\circ} \mathrm{C}$. The samples were then removed from the distilled water and trimmed to a rectangular shape using a low-speed diamond saw (Isomet, Buehler Ltd, Lake Bluff, IL, USA). Notches were made along the dentin-adhesive interface using a diamond bur that was fixed in a low-speed drill press. The samples were cut into hourglass shapes with necks approximately $1 \mathrm{~mm}$ wide. The specimens were then sliced into $0.6-\mathrm{mm}$-thick sections perpendicular to the interface using the low-speed diamond saw.

\section{Microtensile Bond Strength Test}

Each specimen was attached to the testing device (Bencor-Multi-T, Danville Engineering Co, Danville, CA, USA) using a cyanoacrylate adhesive (Scotch Super Glue Gel, 3M, St Paul, MN, USA). The jig was then mounted in a universal testing machine (Lloyd Instrument Amtek Inc, Largo, FL, USA). Tensile force was applied at a crosshead speed of $1 \mathrm{~mm} /$ minute. The maximum load at the point of fracture divided by the cross-sectional surface area of the bonded surface was used to calculate the microtensile bond strength. Twoway ANOVA was performed to evaluate the effects of two experimental factors: the two-step self-etch adhesive that was used and the experimental conditions. The interaction of these two factors on the MTBS was also evaluated. Multiple comparisons were performed post hoc using the least significant difference (LSD) method. Differences with $p$-values $<0.05$ were considered statistically significant.

\section{SEM Evaluation of Fracture Modes}

Five fractured surfaces from each group were examined in order to evaluate fracture modes. Fractured specimens were mounted on stubs, gold sputter-coated (Bio-Rad Polaron Division SEM Coating System, Polaron Instruments Inc, Agawan, MN, USA) and examined under scanning electron microscopy (SEM; S-4700, Hitachi, Tokyo, Japan) at magnifications of 110x and 2,500x. The fracture modes were categorized according to the method used by Sano and others ${ }^{27}$ : mixed (M), adhesive (A), cohesive in resin (CR) and cohesive in tooth (CT). The morphological characteristics of the fracture surfaces were also examined.

\section{RESULTS \\ Microtensile Bond Strength Test}

The results of two-way ANOVA suggested that the MTBS results (Table 2) were significantly influenced by both the self-etch adhesive used $(p<0.0001)$ and the experimental conditions applied $(p<0.0001)$. There was a significant interaction between the two factors $(p=0.003)$.

Multiple comparisons made with a post hoc LSD test revealed that Tyrian SPE yielded significantly lower MTBS than Clearfil SE Bond and AdheSE $(p<0.05)$.

In all three self-etch adhesive groups tested, the control groups showed significantly higher MTBS than most of the contaminated groups in this study. The exceptions were CG1 for Clearfil SE Bond and CG2 and CG3 for Tyrian SPE.

For Clearfil SE Bond, the control group and CG1 had a significantly higher MTBS than CG2, CG3 and CG4 $(p<0.05)$. There were no significant differences in MTBS between the control group and CG1 $(p>0.05)$ or in MTBS between CG2, CG3 and CG4 $(p>0.05)$. For AdheSE, all of the contaminated groups had a significantly lower MTBS compared to the control group $(p<0.05)$. CG2 had a significantly higher MTBS than CG3 $(p<0.05)$. For Tyrian SPE, the control group had a significantly higher MTBS compared to CG1 and CG4 $(p<0.05)$. MTBS for each control and experimental group is illustrated in Table 2.

\section{SEM Evaluation of Fracture Modes and Morphological Characteristics of Fractured Surfaces}

The results of fracture mode analysis showed that adhesive fractures and mixed fractures were prevalent in specimens treated with Clearfil SE Bond and AdheSE. In contrast, adhesive fractures were dominant in the Tyrian SPE group.

For specimens treated with Clearfil SE Bond, the fracture surfaces in the control group (Figure 2A) were characterized by numerous open dentinal tubules and a scaly appearance, but CG2 (Figure 2B) had a "washed-out" appearance. For specimens treated with Tyrian SPE, the fracture surfaces of the control group (Figure 3A) and CG2 (Figure 3B) were similar in appearance, including wide-open dentinal tubules that were partly filled with broken resin tags. For specimens treated with AdheSE, the fracture surfaces of CG1 had blood residues remaining on the fractured surface.

\section{DISCUSSION}

The Tyrian SPE group had a significantly lower MTBS compared to the Clearfil SE Bond and AdheSE group $(p<0.05)$. This result was consistent with previous studies ${ }^{23-25}$ that reported that the MTBS of the 


\begin{tabular}{|c|c|c|c|c|c|}
\hline \multicolumn{2}{|c|}{ Table 2: The Results of Microtensile Bond Strength Test } \\
\hline Source & df & Sum of Squares & Mean Squares & F-value & $p$-value \\
\hline Adhesive * group & 8 & 117160 & 14645 & 2.97 & 0.0033 \\
\hline Group§ & 4 & 431541 & 107885 & 20.77 & $<.0001$ \\
\hline Adhesive§ & 2 & 296331 & 148165 & 28.52 & $<.0001$ \\
\hline $\begin{array}{l}\text { I'Adhesive * Group' means the interaction between the two factors, adhesive (Clearfil SE, AdheSE, and Tyrian SPE) and group (control group and four contamination groups). } \\
\text { §Group includes the control group and four experimental contamination groups. SAdhesive includes three adhesives (Clearfil SE, AdheSE, and Tyrian SPE). }\end{array}$ \\
\hline
\end{tabular}

\begin{tabular}{|c|c|c|c|}
\hline & Clearfil SE & AdhesE & Tyrian SPE \\
\hline Control & $49.34 \pm 8.0^{a}$ & $45.84 \pm 9.8^{\mathrm{a}}$ & $32.08 \pm 10.2^{a}$ \\
\hline Contamination group 1 & $42.00 \pm 8.3^{\mathrm{a}}$ & $26.24 \pm 10.4^{\text {bc }}$ & $23.21 \pm 7.7^{b}$ \\
\hline Contamination group 2 & $34.59 \pm 12.5^{b}$ & $32.95 \pm 15.5^{b}$ & $25.95 \pm 13.6^{\mathrm{ab}}$ \\
\hline Contamination group 3 & $29.14 \pm 9.9^{b}$ & $21.31 \pm 8.5^{c}$ & $25.33 \pm 10.1^{\mathrm{ab}}$ \\
\hline Contamination group 4 & $30.96 \pm 9.5^{\mathrm{b}}$ & $27.09 \pm 16.8^{\mathrm{bc}}$ & $19.13 \pm 9.5^{b}$ \\
\hline
\end{tabular}
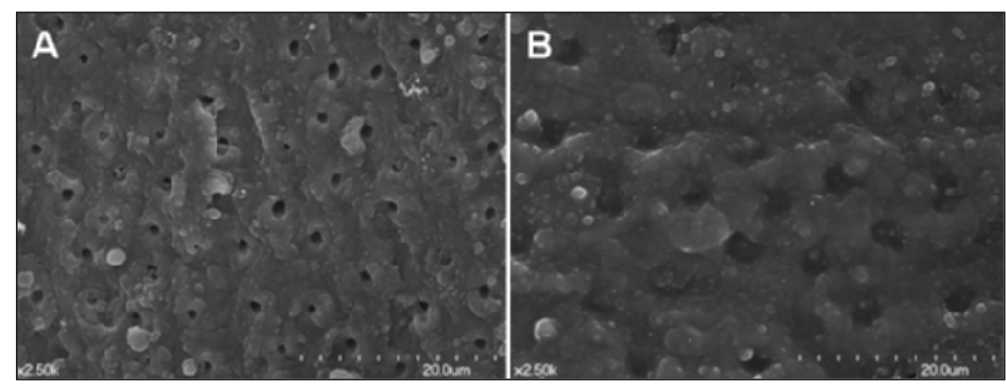

Figure 2. SEM evaluation of fracture surfaces in the control group (2A) and contamination group 2 (2B) of Clearfil SE Bond group (2500x). Numerous open dentinal tubules and a scaly appearance are shown in the Clearfil SE Bond control group (2A). A "washed-out" appearance was observed in the Clearfil SE Bond contamination group 2 (2B).
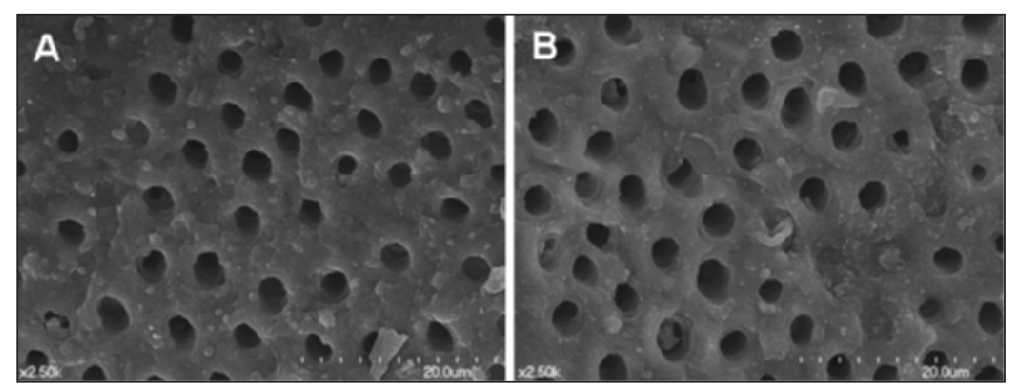

Figure 3. SEM evaluation of the fracture surfaces in the control group (3A) and contamination group 2 (3B) of Tyrian SPE group (2500x). The fracture surfaces had similar characteristics in both groups, including wide-open dentinal tubules and scaly appearances.

Tyrian SPE group was lower than the Clearfil SE Bond group. Although there is no consensus on the relationship between bond strength and acidity of self-etch adhesives, it is generally accepted that aggressive self-etch adhesives, such as Tyrian SPE, result in low bond strengths. ${ }^{24,26}$ This may be attributed to the inhibitory effects of the acidic monomer remaining in the oxygen-inhibited layer, which may inhibit polymerization of the chemical cure resin com- posite due to an acid-base reaction with a tertiary amine. ${ }^{28}$ Although there is a lesser chance for this acid-base reaction to occur in light-cured resin composites because of the fast initiation and production of free radicals, chances exist for this reaction to occur if curing is delayed. There may be a greater chance for this reaction to occur when using Tyrian SPE, because this system contains a greater concentration of acidic monomers compared to mild or moderate self-etching adhesives. ${ }^{28}$ The lower MTBS of the Tyrian SPE group observed in the current study was also consistent with the results of fracture mode analysis, which showed that Tyrian SPE had a much higher proportion of adhesive fractures $(92 \%)$ than Clearfil SE Bond (56\%) and AdheSE (40\%). The lower bond strength of Tyrian SPE may also be explained by the findings of another previous study $^{29}$ reporting that the Tyrian SPE group exhibited irregular and poor hybridization with few resin tags in the hybrid layer, which was in contrast to the AdheSE group that yielded consistent and uniform hybridization with homogeneous resin tags.

The specimens treated with AdheSE and Tyrian SPE, which were rinsed with water after contamination (CG2), exhibited higher bond strengths compared to groups with specimens that were not rinsed (CG1). In contrast, specimens treated with Clearfil SE Bond, which were not rinsed, had higher bond strengths than the group that was rinsed. This may be explained by differences in acidity of the three self-etch adhesives used in this experiment: mild (Clearfil SE Bond), moderate (AdheSE) and strong (Tyrian SPE). ${ }^{22}$ Any water that remains after rinsing may further dilute the weak acid in the primer of the Clearfil SE Bond and therefore result in incomplete infiltration of successive self-etch primers. 
CG3 and CG4 resulted in the lowest MTBS values, specifically CG3 in the Clearfil SE Bond and AdheSE groups and CG4 in the Tyrian SPE group. This suggests that blood contamination results in the most significant decrease of MTBS when it occurs after lightcuring of the adhesive layer. These results agree with the findings of previous studies, ${ }^{4,12}$ which suggested that salivary contamination that occurs after light-curing resulted in a significant decrease in bond strength. The results of the current study also agreed with other previous studies, ${ }^{30-31}$ which reported that adhesives with oxygen-inhibited layers showed significantly higher interfacial bond strengths than adhesives without oxygen-inhibited layers or adhesives contaminated by water or saliva. The lower bond strengths that the authors of the current study observed in CG3 and CG4 may be attributed to loss of the oxygen-inhibited layer, which contained unreacted monomers that were able to improve adhesion between successive layers by the formation of covalent bonds within an interpenetrating network. This mechanism has been suggested by previous investigators. ${ }^{32-33}$

In the current study, MTBS of the contamination groups was lower overall than for that of the control groups. In CG1, blood protein and blood plasma may have adsorbed onto the bonding surfaces, as suggested by Pashley and others, ${ }^{34}$ and this may have inhibited adhesion between the dentin and dental composite. In CG2, the water used for rinsing may not have been completely removed, and the remaining excess water may have adversely affected the bond between dentin and composite. The reduced bond strengths observed in CG3 and CG4 may be partly explained by the loss or thinning of the oxygen-inhibited layer and partly by adsorption of blood proteins onto the bonding surface.

SEM examination revealed characteristic features of each group, which agrees with the results of the MTBS tests. When comparing the Clearfil SE Bond control group (Figure 2A) and CG2 (Figure 2B), CG2 had a "washed-out" appearance, which indicates that the infiltration of primer after rinsing was not as effective as in the control group. This finding may partially explain the lower MTBS observed in CG2 compared to the control group. The ineffective infiltration of additional primers may be related to the further dilution of the weak acidic monomer of Clearfil SE Bond. In contrast, when comparing the Tyrian SPE control group (Figure 3A) and CG2 (Figure 3B), CG2 exhibited very similar fracture surfaces to that of the control group. Both were characterized by wide-open dentinal tubules and a scaly appearance on the fracture surface, indicating that the infiltration of additional primer was almost as effective as in the control group. This finding may partially explain the similar MTBS of CG2 and the control group in specimens treated with Tyrian SPE. The effective infiltration of addi- tional primer of Tyrian SPE may have occurred due to strong acidity of the Tyrian self-etch primer. The AdheSE control group was characterized by dentinal tubules that were well obturated with resin tags. The CG1 of specimens treated with AdheSE exhibited fracture surfaces contaminated with blood residues. This may partially explain the lower MTBS in CG1 compared to that of the control group in specimens treated with AdheSE.

The current study has several limitations, one is that there was no control group in which the bonding procedures were completely repeated from the beginning; for example, by grinding away the blood contaminated part of the dentin, then reinitiating the bonding procedure. A follow-up study with a better experimental design, including the control group mentioned above and a larger number and various other shapes of the specimens, is currently being conducted by the authors to further understand the effects of blood contamination on the bond strengths of two-step self-etch adhesives. Future studies that look for effective decontamination methods are also needed.

\section{CONCLUSIONS}

Within the scope of the current study, the results showed that blood contamination negatively affected the microtensile bond strength of two-step self-etch adhesives to dentin. The null hypothesis was rejected. In addition, neither decontamination method used in this study overcame the effects of blood contamination.

(Received 10 August 2009)

\section{References}

1. Vaidyanathan TK \& Vaidyanathan J (2009) Recent advances in the theory and mechanism of adhesive resin bonding to dentin: A critical review Journal of Biomedical Materials Research Part B: Applied Biomaterials 88(2) 558-578.

2. Yazici AR, Celik C, Ozgunaltay G \& Dayangac B (2007) Bond strength of different adhesive systems to dental hard tissues Operative Dentistry 32(2) 166-172.

3. Kiremitci A, Yalcin F \& Gokalp S (2004) Bonding to enamel and dentin using self-etching adhesive systems Quintessence International 35(5) 367-370.

4. Townsend RD \& Dunn WJ (2004) The effect of saliva contamination on enamel and dentin using a self-etching adhesive Journal of the American Dental Association 135(7) 895901; quiz 1036, 1038.

5. el-Kalla IH \& García-Godoy F (1997) Saliva contamination and bond strength of single-bottle adhesives to enamel and dentin American Journal of Dentistry 10(2) 83-87.

6. Abdalla AI \& Davidson CL (1998) Bonding efficiency and interfacial morphology of one-bottle adhesives to contaminated dentin surfaces American Journal of Dentistry 11(6) 281285. 
7. Hitmi L, Attal JP \& Degrange M (1999) Influence of the timepoint of salivary contamination on dentin shear bond strength of 3 dentin adhesive systems Journal of Adhesive Dentistry 1(3) 219-232.

8. Yoo HM, Oh TS \& Pereira PN (2006) Effect of saliva contamination on the microshear bond strength of one-step self-etching adhesive systems to dentin Operative Dentistry 31(1) 127-134.

9. Ari H, Donmez N \& Belli S (2008) Effect of artificial saliva contamination on bond strength to pulp chamber dentin European Journal of Dentistry 2 86-90.

10. Park JW \& Lee KC (2004) The influence of salivary contamination on shear bond strength of dentin adhesive systems Operative Dentistry 29(4) 437-442.

11. Sattabanasuk V, Shimada Y \& Tagami J (2006) Effects of saliva contamination on dentin bond strength using all-inone adhesives Journal of Adhesive Dentistry 8(5) 311-318.

12. Fritz UB, Finger WJ \& Stean H (1998) Salivary contamination during bonding procedures with a one-bottle adhesive system Quintessence International 29(9) 567-572.

13. Raffaini MS, Gomes-Silva JM, Torres-Mantovani CP, PalmaDibb RG \& Borsatto MC (2008) Effect of blood contamination on the shear bond strength at resin/dentin interface in primary teeth American Journal of Dentistry 21(3) 159-162.

14. Yoo HM \& Pereira PN (2006) Effect of blood contamination with one-step self-etching adhesives on microtensile bond strength to dentin Operative Dentistry 31(6) 660-665.

15. Powers JM, Finger WJ \& Xie J (1995) Bonding of composite resin to contaminated human enamel and dentin Journal of Prosthodontics 4(1) 28-32.

16. Dietrich T, Kraemer M, Losche GM, Wernecke KD \& Roulet $\mathrm{JF}$ (2000) Influence of dentin conditioning and contamination on the marginal integrity of sandwich Class II restorations Operative Dentistry 25(5) 401-410.

17. Pashley DH (1991) Dentin bonding: Overview of the substrate with respect to adhesive material Journal of Esthetic Dentistry 3(2) 46-50.

18. Pashley EL, Tao L, Mackert JR \& Pashley DH (1988) Comparison of in vivo vs in vitro bonding of composite resin to the dentin of canine teeth Journal of Dental Research 67(2) 467-470.

19. Strydom C (2005) Handling protocol of posterior composites rubber dam Journal of the South African Dental Association Journal 60(7) 292-293.

20. van Schalkwyk JH, Botha FS, van der Vyver PJ, de Wet FA \& Botha SJ (2003) Effect of biological contamination on dentine bond strength of adhesive resins Journal of the South African Dental Association Journal 58(4) 143-147.

21. Eiriksson SO, Pereira PN, Swift EJ, Heymann HO \& Sigurdsson A (2004) Effects of blood contamination on resinresin bond strength Dental Materials 20(2) 184-190.
22. Loguercio AD, Moura SK, Pellizzaro A, Dal-Bianco K, Patzlaff RT, Grande RH \& Reis A (2008) Durability of enamel bonding using two-step self-etch systems on ground and unground enamel Operative Dentistry 33(1) 79-88.

23. Kenshima S, Reis A, Uceda-Gomez N, Tancredo Lde L, Filho LE, Nogueira FN \& Loguercio AD (2005) Effect of smear layer thickness and $\mathrm{pH}$ of self-etching adhesive systems on the bond strength and gap formation to dentin Journal of Adhesive Dentistry 7(2) 117-126.

24. Reis A, Grandi V, Carlotto L, Bortoli G, Patzlaff R, Rodrigues Accorinte Mde L \& Dourado Loguercio A (2005) Effect of smear layer thickness and acidity of self-etching solutions on early and long-term bond strength to dentin Journal of Dentistry 33(7) 549-559.

25. Sensi LG, Lopes GC, Monteiro S Jr, Baratieri LN \& Vieira LC (2005) Dentin bond strength of self-etching primers/adhesives Operative Dentistry 30(1) 63-68.

26. Kaaden C, Powers JM, Friedl KH \& Schmalz G (2002) Bond strength of self-etching adhesives to dental hard tissues Clinical Oral Investigations 6(3) 155-160.

27. Kaneshiro AV, Imazato S, Ebisu S, Tanaka S, Tanaka Y \& Sano H (2008) Effects of a self-etching resin coating system to prevent demineralization of root surfaces Dental Materials 24(10) $1420-1427$.

28. Sanares AM, Itthagarun A, King NM, Tay FR \& Pashley DH (2001) Adverse surface interactions between one-bottle lightcured adhesives and chemical-cured composites Dental Materials 17(6) 542-556.

29. Sensi LG, Marson FC, Belli R, Baratieri LN \& Monteiro S Jr (2007) Interfacial morphology of self-etching adhesive systems in dentin Quintessence International 38(2) e112-119.

30. Truffier-Boutry D, Place E, Devaux J \& Leloup G (2003) Interfacial layer characterization in dental composite Journal of Oral Rehabilitation 30(1) 74-77.

31. Kim JS, Choi YH, Cho BH, Son HH, Lee IB, Um CM \& Kim CK (2006) Effect of light-cure time of adhesive resin on the thickness of the oxygen-inhibited layer and the microtensile bond strength to dentin Journal of Biomedical Materials Research Part B: Applied Biomaterials 78(1) 115-123.

32. Vankerckhoven H, Lambrechts P, van Beylen M, Davidson CL \& Vanherle G (1982) Unreacted methacrylate groups on the surfaces of composite resins Journal of Dental Research 61(6) 791-795.

33. Kao EC, Pryor HG \& Johnston WM (1988) Strength of composites repaired by laminating with dissimilar composites Journal of Prosthetic Dentistry 60(3) 328-333.

34. Pashley DH, Nelson R \& Kepler EE (1982) The effects of plasma and salivary constituents on dentin permeability Journal of Dental Research 61(8) 978-981. 